\title{
LOS DORIOS: SU MIGRACION Y SU DIALECTO
}

The author does not share Chadwick's views about the presence of a clower classo Proto-Dorian population in Mycenaean Peloponnesus. Archaeological data appear to be no obstacle against the traditional view of a post-mycenaean Dorian migration from Northern to Southern Greece. Tradition aud Myth are to be credited as reliable sources: the Return of the Heraclidae is in fact a true account for low and when the Dorians occupied Southern Greece.

The so-called ndifférences clialectalesn in Mycenaean cannot be interpreted as evidence of a proto-Doric speaking population: the uspecial Mycenaean is by no means proto-Doric; between Mycenaean (and closely related dialects) and (proto-)Doric there are many early and late differences and divergent developments. It turns out quite difficult to believe that Mycenaeans and Proto-Dorians met in the Peloponnesus. Differences should have been eliminated and, instead of two divergent developments, there should be expected a common development and only one. The author thinks that the $\tau_{1}>\sigma_{1}$ isogloss provides a decisive factor to invalidate Chadwick's viervs.

En La Parola del Passato (enero-febrero 1976), pp. 103-I17, con el título "Who were the Dorians?", publica Cliadwick el texto de una conferencia leída en Roma unos meses antes; se esté o no de acuerdo con el micenólogo inglés, hay que agradecerle este texto, que nos replantea a fondo la cuestión entera de la fragmentación e interrelación dialectal del griego antiguo. En las páginas que siguen vamos a exponer algunas dudas que nos impiden aceptar la tesis de Chadivick.

I. Euvidentemente el fácil esquema de las tres migraciones y los tres grupos dialectales principales se ha venido abajo; en especial, la descripción del colapso micénico por obra de los dorios, rudos guerreros superiores por sus armas de hierro, entra ya de lleno en lo que Crossland califica de "lurid fantasies»: lo "ben trovato" llevaba demasiada ventaja a lo "vero" y era incluso sospechoso de responder al tópico de que toda civilización llega a un punto en el que no le queda sino "esperar a los bárbaros». De todos modos sigue en pie la verdad de que Cnoso, Pilo y Micenas tuvieron un final tan trágico para ellas como afortunado para los micenólogos, y no han perdido su fuerza impresiva las angus- 
tias cle Pilo (Ventris y Chadwick 1973, 1). 1,37-13\$) ante las ameuazas de un atarue por mar de cuyos protagonistas ya no estamos hoy tan seguros. El colapso de la civilización micénica en Pilo y Micenas es una realididt, en Cnoso una realidad... y un misterio, si mantenemos la fecha de c. I400; también es real que tras ese colapso - pero quizá 110 de forma inmediata y directa - sobreviene una inversión total de la situación lingüística del Peloponeso, aunque parezca incompatible con que "the coming of the Dorians is, archaeologically speaking, a nonevent». Habrá que recordar que, en primer lugar, ya no es opinión común que la presencia doria en el Peloponeso sea la causa del agotamiento micénico, y ni siquiera de fecha coincidente o inmediata con él; lıabrí que recordar también que no siempre es cierto que cambio de lengua y cambio de población impliquen cambio cultural, y más en este caso, sabido que hay relación entre la Hélade del Norte, invasora, y la del Sur, invadida y netamente superior en lo cultural, incluso como para producirse ya un primer ensayo del tópico "Craecia capta». Además, no hay cambio en cuanto aparición de elementos culturales nuevos, en cuanto ruptura, pero sí en cuanto empobrecimiento u oscurecimiento de los antiguos. Importa poco que el movimiento dorio fuera o no el agente destructor $o$, simplemente, ocupante de un vacío y recuperador de una discontinuidad: entiendo, con Bengtson 1960, p. 52, que negar el movimiento dorio desile el Norte hacia el Peloponeso, Creta y las Cícladas es "ine arge Verirrung der modernen IIyperkritik" y entendería, no simplemente por concesiones eclecticistas, la presencia de elcmentos dorios en el Peloponeso antes de c. r200, pero sin que fuesen significativos, especialmente en lo que a la lengua se refiere; y de admitir tal presencia doria no pasaría, salvo documentación expresa muy clara, a concluir que la relación entre dominantes y dominados pudiese establecerse en términos de estirpes, pues ni siquiera la establecería en términos de griegos / no griegos, bastaindome para cllo el considerar la antroponimia micénicia y del mito y de la épica, ya sin entrar en el conteniclo de estos dos últimos.

2. Quizá lo más siguificativo de la lengua de las tablillas para nuestra Dialectologia ha sicho remachar que la isoglosa principal es la que divide lescle antes de c. I 400 el gricgo comun en clos: el (que mantiene $t \mathrm{y}$ th ante $i$ (dorio, noroccidental, tesalio y beocio) y el que palatalizó ambas oclusivas (micénico, arcadio-chipriota, jónico-ático y lesbio). No podría concordar con Cliadwick en admitir que los dorios estuviesen ya en el Peloponeso en la fecha en que se produjo este cambio fonético porque no veo la forma de hacer una distrilución del territorio 
que separase a dorios y no dorios, y menos aún veo que, de convivir unos y otros, se diesen las condiciones sociopoliticas que produjesen esa especie de $\delta ı \gamma \lambda \omega \sigma \sigma i \alpha$ que nos explicase por qué la lengua $B$ o nivel inferior no conoció esa evolución fonética. Habrá que precisar que el problema 110 se soluciona con sacar a unos y otros del Peloponeso, incluso de la Hélade, y darles cualquier otro asentamiento en cualquier otra

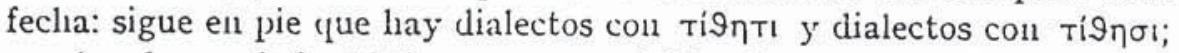
en el orden sociolingüístico parece excluído el argumento de que quienes palatalizaron $l$ y $t h$ ante $i$ eran una pequeña "Herrenschicht" reducida a las fortalezas y apenas comunicada con la población sometida.

Entiendo que para explicar que tesalio, beocio y griego occidental $n$ conocen el proceso de palatalización de $t$ y th no hay otra solución plausible que trazar una frontera que será los golfos Calidonio $y$ de Corinto, y la Cadmeida (luego Beocia: Thuc. I I2, 3): al Norte, los que mantuvieron ambas dentales, $\mathrm{y}$ al Sur, coincidiendo plenamente con el ámbito de la civilización micénica, los que las asibilaron. Del caso del lesbio hablaremos más adelante, aunque ja señalemos que no identificamos sin más nuestra posición con la afirmación tucidídea de que ya

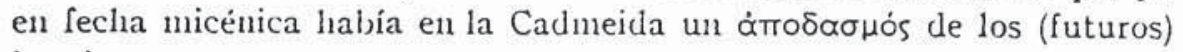
beocios.

3. Pasa Chadwick a continuación a considerar el valor de las tradiciones y las relega a "only secondary suurces" frente a las pruebas arqueológicas y lingüísticas. Bien está tal prevención, porque sabemos el escaso valor que puede tener una tradición oscurecida en su transmisión de generación en generación, o manipulada descaradanente para servir a fines de propaganda y reivindicación politicas, pero también estará de más el hipercriticismo, sobre todo si se quiere distinguir entre tradición (épica, local, etc.) y noticia histúrica, ya que esta última en la mayoría de los casos no ha de ser más que una tradición a la que el espíritu crítico de Heródoto, Tucídides, Pausanias..., lian dado por buena y fiable... y pasado de Caliope o cualquier otra a Clío.

Ein nuestro casu concreto el "returno de los Heraclidas" parece tradición histórica fiable, real, aunque manipulada; no confirmacla ni desmentida por la Arqueología, pero confirmada plenamente por la Linguística, y utilizable por tanto en la descripción histórica (cf. Desborough y Hammond 1964, p. 3r-35 y Desborough 1964, p. 244 y ss.). De este retorno sabemos a través de Tucidides, Pausanias, Heródoto, Helánico, etc.: es importante señalar que todas las informaciones hablan de returno de Heraclidas $y$ no de dorios, y que se distingue a unos de otros claramente: Tirteo fr. 2 West y. Tucídicles I I2, , 
se limitan a presentarnos a los Heraclidas en instrumental comitativo con los dorios; menos valor asignaríanos al pasaje homérico en que se nos habla del aqueo $\mathrm{y}$ heraclida Tlepólemo, combinado con aquel otro que opone a dorios $y$ aqueos en Creta: que los Heraclidas no eran dorios lo sabian muy bien los espartanos (por ejemplo, Hdt. V 72,3 y otros pasajes); por otra parte, la tradición habla de llegada y no de retorno de los dorios al Peloponeso, detallándose incluso el punto de partida del movimiento (cf. Tirteo fr. 2 West), y es de sospechar que, si los dorios tuviesen conciencia de estar recuperando lo perdido o haciéndose dueños del suelo en que ya vivían como "lower class", la tradición no dejaría de mencionarlo; si se llegara a considerar dorios a los Heraclidas y a su héroe, perdería fuerza la identificación de los dorios con la "lower class" o población sometida en el Peloponeso micénico.

Si añadimos ahora el fr. 9 West de Mimnermo sobre el origen pilio de los colofonios, Heródoto I 56 sobre la Driópide como asentamiento y punto de partida de los dorios, Pausanias II 37,3 sobre que en época predoria Argos y Atenas tenían la misma lengua, Heródoto VIII 73 sobre la presencia jonia en Cinuria, y otras muchas noticias similares (cf. Schwyzer DGE, Appendix III), se verá que hay poco sitio para los protodorios y mucho para los protojonios en el Peloponeso micénico. Y habría que explicar, de admitir la tesis de Chadivick, por qué Arcadia mantiene su dialecto no dorio $y$ sin dorismos considerables y de fecha antigua: en el esquema que nos propone el profesor inglés sería de esperar la presencia plena de dorios en Arcadia, con el consiguiente reflejo lingüístico, ya que, admitidos esos protodorios «lower class» en el Peloponeso micénico, no se entiende muy bien que no hubicran estado y continuaran asentados en la región más pobre; al contrario, admitida la invasión doria tras el colapso micénico, se explica perfectamente la marginación de los arcadios y de su lengua por no haber interesado a los invasores esa región pobre y de dificil acceso.

Si además la Arqueologia está hoy en la opinión de que la civilización micénica no fue destruída por los clorios, sino por otros agentes, o que decayó por sí misma, incluso tal vez por causas naturales y muy probablemente por luchas internas (cf. Thuc. I I2, 2), habiéndose limitaclo los dorios a aprovechar tal decadencia, resultará más clara la realidad del "retorno de los Heraclidas", apropiado y manipulado por los dorios para justificar su presencia y sus conquistas (cf., por ejemplo, Carpenter 1966, p. 27 y ss., Desborough 1964 , p. 241 y ss., Desborough y Haminond 1964 , p. 3i y ss.). 
4. Para Chadwick el traer a los dorios desde el Norte al Peloponeso es poco creíble porque para que éste cambiase totalmente su lengua por obra de los recién llegados, éstos habrían de ser "not merely dominant, but numerous», y el Noroeste de Grecia no tenía espacio suficiente para albergar las decenas de miles de hombres que Cliadwick estima . necesarios, ni da muestras de haber sufrido despoblación por emigración. En principio, estoy de acuerdo con postular un número alto de inmigrantes para que su lengua pueda imponerse, pero donde quizá disentimos es en la apreciación de qué número puede considerarse ya alto y suficiente; por ejemplo, me bastaria, si merece algún crédito, la cifra de dos mil que para los espartanos da Isócrates, Panath. 255, y la multiplicaria por diez o quince para el total de los dorios que invadieron el Peloponeso.

Por otra parte, hay que tener en cuenta que invasores e invadidos no hablan lenguas distintas, sino dialectos de una misma lengua, lo que favorece, creennos, la imposición del dialecto invasor aunque sus hablantes sean muchos menos que los del invadido; y, si añadimos que los dialectos griegos de época micénica estaban más próximos entre sí que los de época histórica, casi concluiríamos que la imposición del dialecto protodorio no fue mucho más que un cambio de "norma".

Además, si bien admitimos una invasión numerosa, no estará fuera de lugar recordar que Lingüistica y Arqueología disponen de modelos, construidos sobre experiencias reales, que autorizan la posibilidad de que una minoría fuerte - dicho sea sin concesiones a las slurid fantasicsi) de que hablábamos antes- imponga su lengua (cf. Porzig 1954, 1p. 6r y ss. con ejemplos indoeuropeos). Añádase que tenemos testimonios fiables de que la invasión doria aceleró o produjo la migración de la población invadida, lo cual significa menor resistencia a la imposición del dialecto dorio y, suponemos, podrá atenuar el escepticismo de Chadwick.

Más todavía: si hemos entendido bieu a Hammond 1973, pp. 36-46, y a los arqueólogos y prehistoriadores que presentan los movimientos de los pueblos indoeuropeos -en especial, ilirios, Briges o Brygi, tracios, etc.-, deduciremos que el despoblamiento del Norte y Noroeste de la Hélade no se ha dado y que el movimiento dorio hacia el Sur se debe a la presión de esos otros pueblos, que pasan a ocupar el espacio que les dejan libre dorios, noroccidentales y eolios; recuérdese la presión de los dorios sobre los beocios, a los que también empujan hacia su asentamiento definitivo los tesalios, a su vez empujados por poblaciones noroccidentales. 
Por último, habría que matizar la afirmación de que en "a very short time" se expandieron "vigorously" por Creta y demás islas. Lil caso de Creta es aparte $y$ nos referiremos a él mís adelante. I a expansión por Melo, Tera y el Dodecaneso no parece problema grave, y tal vez. entre ella y la invasión del Peloponeso hay más de un siglo largo, y hasta. tres siglos para varias islas y/0 ciudacles (cf. Cook I964, pp. 18-24).

5. Reparemos además que entre la invasión doria y la documentación epigráfica hay varios siglos que pueden dar cuenta de la desaparición total - salvo casos aislados de hechos de sustrato- de la lengua de la población invadida; tal vez no sea gratuíto suponer que, si clispusiéramos de inscripciones ya para los siglos $\mathrm{xr}$ y siguientes, comprobariamos que la imposición del dialecto invasor no pudo ser repentina ni quizá fue rápida. Recorclemos también que la gran mayoria de los textos epigráficos $-y$ desde luego los de documentación clave para la Dialectología - pertenecen al «Amtsstil», a lenguas y niveles de lengua más bien sujetos a una norma conservadora, a redacción oficial $y$ formularia poco propensa a reflejar la verdadera realidad y varicdad lingüistica. ¿Seria muty disparatado imaginar que las innovaciones postmicénicas que unen dorio y jónico-ático pueden deberse, además de a ser limitrofes, a que bajo la población y el dialecto dorio del Peloponeso hay una población $y$ un dialecto sometidos que, si no lo son, tienen mucho o toclo que ver con los jonios?

6. En el punto 2 hicimos nuestras reservas a que los dorios, si hubiesen estado en el peloponeso ya en fecha micénica, no habrian siclo afectados por la palatalización $\mathrm{TI}, 91>\sigma 1$, pues no vemos razones de orden geo- o sociolingüístico que 1o justifiquen; luego añadiremos otros hechos de no convergencia $y$ de divergencia entre los dialectos dorios y no dorios que nos reafirman en nuestras reservas; y queremos insistir en que, cualquiera que sea la fecha y el lugar en que se produjo la palatalización mencionada, tiene que haber una separación real y efectiva entre quienes se vieron afectados por ella y quienes no.

Con palabras del mismo Chadwick, de ser cierta su hipótesis: «it would be quite impossible for the two dialects to develop independently: each would inevitably influence the othern. Listamos plenamente de acuerdo en la literaliclad $y$ en el conteniclo de esta afirmación, pero creemus que no es una, sino dos, y muy distintas: "to devclop independently" es una imposibilidad que está muy lejos de ser (cuasi) sinónima de "each... influence the othern. Ein efecto, las difercncias $y$ vacilaciones dialectales que presentan las tablillas pollían ser interpretadas como el inevi- 
table influjo o mezcla entre sistemas conviventes, pero eso no es lo mismo que evolución codependiente; ésta se daría si uno y otro sistema participasen en las mismas evoluciones fonológicas, eligiesen, eliminasen, creasen..., unos mismos significantes para unos mismos significados, y ello tanto en el plano morfológico como en el léxico.

$\mathrm{X}$ la evidencia es que entre micénico (con sus más próximos parientes) y (proto)dorio hay divergencias radicales: bien diferencias antiguas que clebieran haber sido borradas por convivencia y evolución codependiente o única, bien diferencias surgidas de que en la fecha de la presunta convivencia cada uno de los sistemas haya tenido evoluciones divergentes que, repetimos, excluyen la convivencia de sus respectivos hablantes. l'or tanto, pucde - más bien, podria- haber influjo o mezcla derivada de contacto, pero eso no es evolución común de sistemas por convivencia de sus hablantes. Por supuesto 1:o importa ni a Chadwick ni a mí la presencia ocasional y aislada de algun(os) escriba(s) protodorio(s): una golondrina no hace verano.

Sin entrar en la discusión de que las variantes o diferencias del micénico puedan ser interpretadas como protodorismos, nos interesa dejar claro ya que admitimos la posibiliclad cle que en un sistema lingüístico Jaya diferencias o vacilaciones que, dejando de lado cuestiones de orden diacrónicos, no necesiten ser explicadas como resultado de sustrato, convergencia, mezcla, sincretismo, etc. Un dialecto no es una variante espacial y temporal de un sistema en la que queden excluidos sin más la variante fonética y fonológica, el alomorfismo libre o condicionado, la sinoninia, etc.

1.1 lector perdonari nuestra insistencia en que no es lo mismo que $A$ inlluya sobre 13 y que $\Lambda$ y b tengan evoluciones comunes o, mejor dicho, una única evolución común. X después de este fárrago teórico, pasaremos al análisis de hechos concretos aducidos por Chadwick, hechos que no representan la nivelación de diferencias dialectales, sino la mezcla que deja subsistir la diferencia que impide hablar de convivencia.

7. Cliadwick aprovecha el estudio de Risch ng66 sobre las diferencias dialectales en el micénico y sugiere que el especial awas in fact protoDoric». Antes de entrar en el análisis detallado de tales y otras diferencias, vamos a precisar que, por muy atractiva que resulte la tesis de Risch, micénico "normal" y "especial" no tienen por qué ser necesariamente dos dialectos o niveles diferentes, y es posible que los rasgos que definen al "normaln sean vacilaciones o variantes dentro de un micénico único; esta precisión previa no prejuzga que el análisis de las diferencias nos lleve a concluir que algunas de ellas no son ni siquiera 
relevantes como tales ni como criterio de clasificación y articulación de los dialectos:

I) El uso de $-i$ en lugar de $-\epsilon(=-e i)$ en el dativo singular de los temas consonánticos. P’arece opinión común que $-i$ es en origen desinencia de locativo y $-e i$ lo es de dativo; más que de diferencia dialectal puede tratarse de las primeras manifestaciones de un proceso común - sincretismo de dativo, instrumental y locativo - que, a mayor o menor ritmo, se ha cumplido en todos los dialectos, con selección de la forma $-i$ para los temas que nos ocupan, pero que en los neutros en - $\alpha s$

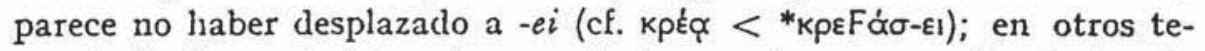
mas todavía en el siglo $\mathrm{v}$ hay vacilaciones e inconsecuencias dentro de un mismo dialecto, por ejemplo, en ático con plurales -āoı $(-\eta \sigma \iota) /-\bar{\alpha} ı \sigma ı$

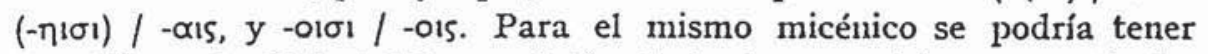
por diferencia dialectal la aparición de hechos paralelos, como duales en -opi frente a $-o$ y procesos similares de analogía, con o sin reflejo en el griego del primer milenio.

Sabemos que estas presuntas o reales diferencias dialectales van ligadas a las "manos", a los escribas, y que en Pilo y Micenas hay alguna (mano" que sinultanea $-i$ con $-e i$, lo que obliga a concluir, si seguimos a Chadwick, que se trata de escribas (proto)dorios que han de utilizar el dialecto oficial o standard, pero hacen concesiones más o menos conscientes a su propio dialecto, pues sería impensable la situación inversa: que el escriba no dorio diese entrada a formas dorias; si tal cosa es verdad, extraña grandemente que los dorismos o protodorismos de las tablillas no sean más abundantes $y$, sobre todo, más claros, por ejemplo

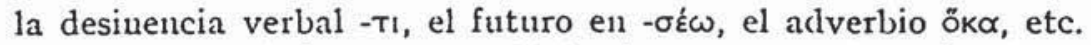

Añade Chadwick: "I can think of no reason why this change" - la sustitución de $-e i$ por $-i$ - "should have begun in the P'roto-Doric dialect: but equally there is no reason why, if it did, that should not have been the source from which it spread to the rest of Greecen: sin duda esta consideración tiene más de "vishful thinking" que de argunento y no le apoya mucho la sugerencia por Risch de que en arcadio -evidentemente muy próximo al micénico "normal" (y yo añadiría que al "especialn, al micénico, lisa y llanamente) - también es reciente la sustitución de $-\epsilon 1$ por -1 , pues lo que ocurre aquí puede entenderse muy bien como la consumación tardía de un proceso común en un ámbito dialectal marginal $\mathrm{y}^{r}$ conservador, es decir, algo similar a lo que en arcadio $y$ otros dialectos octurre con la eliminación de $F$, la pérclicla del número dual, etc.

2) Chaclwick no toma en consideración la quie entendemos como más significativa de las diferencias estucliaclas por Risch: nuicénico normal 
dipa, atimite, timitija..., frente a especial atemito, etc. y griego común

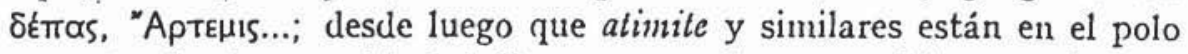

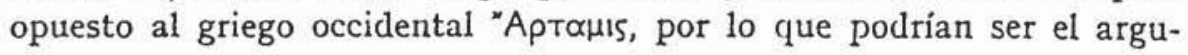
mento más válido, pero todavía insuficiente, y más si la forma "Apт๔uıs es de fecha postnicénica. Y sin ánimo de negar por negar cuanto se opone a nuestro punto de vista, apuntamos que $-i$ - y $-e$ - pueden ser simples vacilaciones ortográficas para reflejar una realización muy cerrada del fonema $|\check{e}|$ en contexto 'ante labial', realización que la norma (orto)gráfica evita en la generalidad de los casos.

3) Entiendo que el problema de la evolución de las líquidas y nasales en función sonántica debe estudiarse atendiendo a los hechos reales y sin pretender que encajen en esquemas preconcebidos de regularidad y compartimentación dialectal rígida; hay tal regularidad, pero más compleja de lo que a primera vista parece. En cualquier dialecto del primer milenio hay vacilaciones o inconsecuencias que no se explican, salvo casos muy concretos, con el récurso al préstamo, al sustrato, a la analogía..., sino que hay que echar mano de criterios como los de contexto fono y morfológico, hay que tener en cuenta que la tensión entre uno y otro puede originar dobletes, hay que contar con que en las evoluciones actuan tendencias que pueden desembocar en la regularidad absoluta o dejar subsistir variantes que la lengua eliminará o podrá mantener, incluso aprovechándolas morfológica o lexicalmente. El lector nos disculpará de tratar aquí el tema por extenso y nos remitiremos a Adraclos $195^{8}$ y I969, Risch I966, Morpurgo I968, Bader 1970 y Moralejo I973a. Eintendemos que:

a) Iin micénico se ha producido ya el desarrollo pleno y fonologización de la vocal de apoyó de la sonante. Está bien documentado el timbre $o$, pero también el timbre $a$, siendo improcedente tenerlo por ajeno al dialecto, y más improcedente todavía el negarse a reconocer lecturas que obliguen a admitirlo. La coexistencia de ambos timbres no nos parece apoyo a la tesis de la koıń micénica.

b) Lin jónico-ático y en griego occidental hay documentación mayoritaria clel timbre $a$, pero no faltan ejemplos cle $o$, incluso en elenentos centrales de la Morfología y del I,éxico; en eólico y en arcadio-chipriota es $o$ el timbre mayoritario, pero sin que falte $a$ en iguales condiciones (uue $o$ en jönico-ático y occidental.

c) Iil contexto fónico parcce influir por cuauto guturales, labiales y $u$ semiconsonántica puclen favorecer la aparición del timbre $0, \mathrm{y}$ también $u$; la proximidad de consonantes anteriores puede propiciar un timbre $i$. 
d) Contexto fónico y morfológico pueclen complementarse o contradecirse, sin duda por la imposición de analogías que pueden tener puntos de partida $y$ direcciones opuestos. Aqui no son de relevancia las fronteras dialectales, por lo que 110 procecle tachar de préstano o sustrato lo que no encaje en la norna general, ni se puede ver mezclas en la existencia de clobletes.

Bajando a los datos concretos, ni $\gamma$ pó $\phi \omega$ y afines son eolismos en

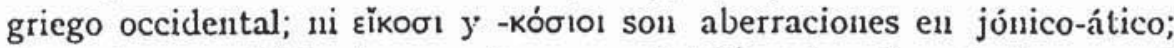
ui son incompatibles pemo y pema en micénico; ni es de extrañar que el griego posterior tenga solamente $\sigma \pi \varepsilon \dot{p} \mu \alpha$, pero $\alpha i \mu o-y$ $\alpha i \mu \alpha-$ en compo-

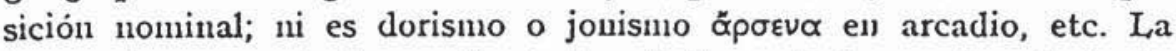

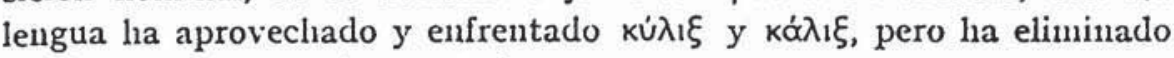
las formas en - $-\mu$ o e impuesto las en - $\mu \alpha$.

Aplicado todo lo anterior al micénico y a la tesis de Chadivick, habrá que repetir que las variantes que detectamos no son indicio de mezcla dialectal y que, como el mismo Cliadwick reconoce, un timbre $a$ (que fuese) anómalo en micénico más llevaria a pensar en el jónico que en el dórico. Lin definitiva, que el micéuico presente variantes demasiado chocantes no es de extrañar, si atendemos a su fecha y suponemos que los dialectos posteriores han dispuesto de varios siglos para eliminar o conservar esas mismas $\mathrm{y} / \mathrm{u}$ otras variantes, según el rendimiento que tuvieran en el plano morfológico y en el léxico-semántico.

8. Concuerdo plenamente con Chadivick en que es "even more striking" la preservación de $-t i$ - en algunas formas micénicas. Vamos a clejar de lado las de interpretación dudosa y examinar las seguras: tenemos el étnico tinacasijo, tinivasija, pero tintoalijao, con -si- y -tique son, respectivanente, evolución y conservación de $-l(h) i-$, y también miralijat, para el que es segura la base Mi $\lambda \bar{\alpha}$ Tos. Quisicra poner a consideración del lector que sobre una base en $-(n) l(h) o s$, cuya dental 110 puede sufrir asibilación, el adjetivo étnico derivado será con forma $-(u)$ sios si es propia de un dialecto con $t(h) i>s i$, y con independencia de que el topúnimo y el étnico estén y sean de esc ámbito dialectal o de otro que no altera el grupo $/(h) i$; pero caben otras posibiliclades:

a) Que el étnico sea - por la razón que procecla- posterior a la asibilación: tendrá, suponcmos, $-l(h) i-;$ suponemos, no afirmamos, porque admitimos que las formas con -si- pucden arrastrarle.

b) Que sobre la base -tos, -nthos se recree el étnico con -t-analógicamente y que la forma nueva conviva con la antigua y regular con $-s-$.

c) Que, si el topónimo base y el étnico derivado no pertenecen al ámbito dialectal con $-s i$, la forma con $-t(h) i$ - conservada y originaria 
sea la normal, incluso para quienes palatalizaron $l(h)$, sin que se excluya que éstos, a su vez, dispongan de las formas con -s-.

Iijemplos concretos de lo que propongo pueden ser Boıwría y BoíTios; en micénico hay korisijo, pero el griego posterior sólo conoce Kopiv9ıos, rehecho sobre Kópiv९os, sin que sea necesario razonar que se deba a que en dorio no liubo $\Omega_{1}>\sigma_{1}$; sabemos que formas como

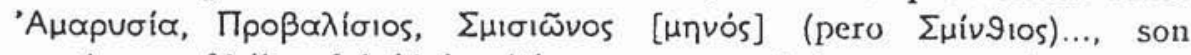
arcaismos, fósiles del jónico-ático, que recreó formas en -९los; otro tanto ocurrió con formas en -Tios, reclucidas a un número escaso frente a las originarias en - $\sigma 105$ (cf. Lejeune r968, pp. 226-229). Cabe, pues, la posibilidad de que mic. miratija, tinneatijao y otros casos paralelos (por ejemplo, kapatija, paratijo, etc.; cf. pp. I62-163 de Landau r958)

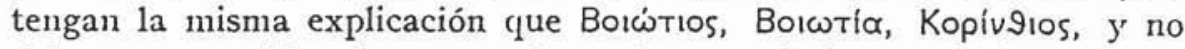
deban ser considerados necesariamente protodorismos ni formas aberrantes dentro del micénico mismo.

Podría reflexionarse también que en topónimos, étnicos y antropónimos puede haber tendencias conservadoras, tendencias a poseer una forma fija y resistente al cambio, con lo que tendría fácil explicación la existencia de dobletes.

Para meritijo, adjetivo formado sobre un tema en -t- ya documentado en las tablillas (gen. sg. merito en $P Y \mathrm{I}_{7 \mathrm{I}}=U n$ 7r 8 ) puede pensarse en una refección de ${ }^{*}$ melisios sobre melit-, paralela a la posterior

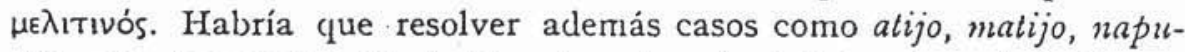
tijo, que, de seguir a Chadwick, plagarían el micénico de protodorismos, y de dorismos el griego común del primer milenio (vid. materiales en Landau I958, Lejeune 1968 y Chantraine r933, pp. 275-289, p. 83, etc.).

No excluímos la posibilidad de que los nombres propios tatiqowen y olinawo sean de individuos protodorios, y ni siquiera aduciríamos pares homéricos como 'Optỉoxos / 'Opoỉoxos. No nos parece ni siquiera discutible la presencia de (proto)dorios en el Peloponeso micénico, pero sí negamos que tal presencia fuese notable $y$, sobre todo, reflejada en la lengua de las tablillas. Ahora bien, para otinawo y tatiqoieen hay explicación suficiente sin echar mano de los (proto)dorios, y a lo expuesto más arriba insistimos en añadir el carácter "volontiers archaisant des anthroponymes" (Lejeune I968, p. 226).

9. Lintre los dialectos dórico y júnico-ático hay divergencias de fecha antigua, y otro tanto entre arcadio-chipriota y dórico; al contrario, las divergencias entre jónico-ático y arcaclio-chipriota son más bien recientes, al igual que las aproximaciones del dórico al jónico-ático. Arcadio-chipriota y jónico-ático son hasta hoy los únicos dialectos 
históricos con pretensiones justas a emparentarse con el micénico, ya sea como continuadores directos de él, ya como indirectos, si se admite, con Heubeck $196 \mathrm{r}$, que la lengua de las tablinlas no se continúa cu ningún dialecto posterior; es clecir, la situación lingüística del Peloponeso $y$ del Atica en fecha micénica puccle presentarse con un protoarcadio muy próximo, si no igual, a un protojonio, y ambos muy próximos, si no iguales, al micénico; si iguales, está claro que la lengua de las tablillas es protojónico-arcadio, o protoarcadio muy próximo al protojonio.

La posibilidad de que los (proto)dorios estuviesen en el Peloponeso ya antes de la destrucción de Pilo y Micenas no parece lingüísticamente viable; acabamos de ver que los presuntos protodorismos de las tablillas pueden tener otras interpretaciones más autoconsecuentes $\mathrm{y}$ sin salirse de la lengua micénica, o - tal es el caso de las sonantes- ni siquiera son invocables como criterio de articulación dialectal, nuáxime si se entienden los dialectos como compartimentos estancos dentro de los cuales está vedada la variedad. Insistimos en que no vemos los con:dicionamientos y las circunstancias que permitan plantear una situación diglósica o un reparto de territorio que expliquen por qué hay mezcla de dorio y no dorio, pero no por qué el dorio no participa en los cambios que afectan a jónico-ático y arcadio-chipriota, y por qué no se han borrado las diferencias antiguas entre estos últimos y el dorio. Añadiria que los protodorismos del micénico habrían de ser más en cantidad y más definitorios y convincentes por su cualidad; entiendo que el único válido, pero bien discutible, es la vacilación $-l i$ - $/-s i$ - y que las tablillas ofrecen ocasión sobrada para que se manifiesten otros, pero no lo hacen. Por otra parte, de los rasgos que definen al micénico normal hay que decir que:

a) el dativo en ei tiene más de elemento común que es eliminado en proceso también común que de característica dialectal; todavía en época histórica hay huellas de él en varios dialectos, tanto en la flexión como en formas fósiles que son primer término de compuestos $\left(\Delta_{1} F\right.$ Ei-

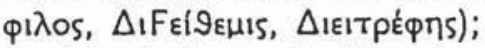

b) de la vacilación $a / 0$ en el timbre de la vocal desarrollada por líquidas y nasales en función sonántica ya hemos dicho que ni siquiera la tenemos por criterio decisivo de "Dialektgliederung»; la única forma llamativa del micénico, forma sin descendencia directa, es pemo, pero recuérdese que tal vocalización seguirá bien documentada en ớno-, en $\dot{\alpha} \lambda \varepsilon 1 \varphi \circ-, y$, para las líquidas en $\mu \bar{\alpha} \mathrm{T} p \circ-$, etc.;

c) solamente dipa, timitija..., carecen de continuadores directos y se oponen al conjunto del griego posterior. 
Lo que define al micénico "especial» resulta ser:

a) en el caso del dativo en $-i$ una manifestación temprana del proceso de sincretismo común a todos los dialectos, no siendo ni siquiera especial que en las tablillas convivan $-e i$ e $-i$;

b) en el caso de pema, una regularización, también común, que elimina de la flexión un tipo -mo, -molos que hubiera sido posible en cualquier dialecto, y relega la vocalización o a primeros términos de compuesto, según acalsamos de ver;

c) en el caso de temilija, etc., también algo común, con la precisa excepción del dórico "Aptauıs, pero quizá no del protodórico.

Nos parece que estos tres rasgos "especiales» e incluso otros, por ejemplo la disimilación de labiovelares, no son prueba inequívoca ni de mezcla dialectal o de diglosia, frente a los "normales", ni de que el micénico no tenga continuadores directos porque lo "normal" o lo específico no se encuentre en la documentación del primer milenio.

Io. Creo que, si en el Peloponeso micénico hubiesen convivido protodorios con protojonios y protoarcadios, necesariamente tendrían que haberse borrado diferencias de fecha prenicénica o micénica, además de liaber participado los protodorios en cambios que, sin duda, son de fecha micénica, empezando por el fundamentalisimo de la asibilación de $t(h) i$; entre las diferencias de fecha antigua que la presunta convivencia debiera haber borrado:

a) Jon.-at. öøos, tóøos, coincidente con arcadio, y ambos opues-

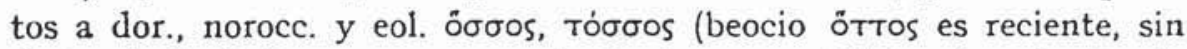
duda, de la fecha en que surge la isoglosa - Tr- ática y beocia, frente a $-\sigma \sigma-$ común); adviértase la coincidencia de griego occidental y eolio. Para la explicación de la divergencia nos remitimos a Risch I955, pp. 6667.

b) Jon.-at. y arcad. - $\nu \propto \alpha$ frente a otra vez dor., norocc. y eol. - $\mu \varepsilon v$ en el infinitivo; lesb. - $\mu \varepsilon v \propto$ a parece innovación.

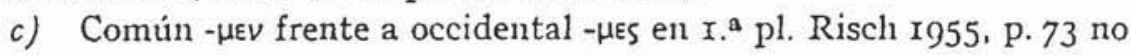
fecha esta elección; concuerdo con López Lire r 974, p. 264 y Bartoněk r9721), pp. 337-338 en que debe de ser antigua, del segundo milenio.

d) Jon.-at. y arcad. (y lesb.) con grado $o$ frente a gr. occ., tes.

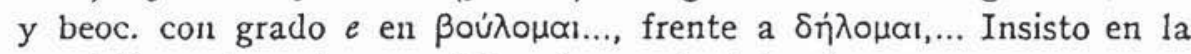
oposición entre griego meridional y septentrional.

Creo que bastarán estos cinco hechos, todos bien presentes y frecuentes en el sistema y en el uso de la lengua, y ya no hará falta recurrir a otros, por ejemplo, el futuro dor. $\delta \omega \sigma \varepsilon ́ \omega$ (con algún ejemplo aislado fuera de ese ámbito dialectal) y que debe de ser más arcaísmo que 
innovación; o a los adverbios temporales, para los que el panorama

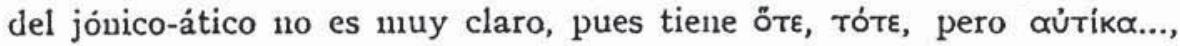
aunque sigue habiendo un enfrentamiento claro de ınicénico, jónicoático $\mathrm{y}$ arcadio-chipriota al griego occidental. Habría que discutir además otras diferencias significativas y que bien pueclen ser ya premicé-

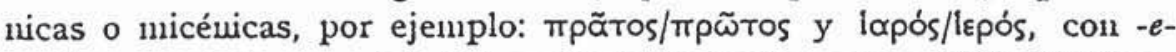

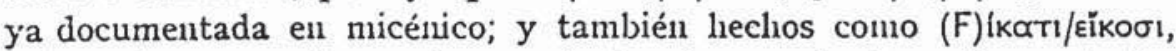
-kótiol frente a -kóoıol, etc.: se trata siempre de elementos centrales - en el sistema y en el uso- de la lengua, para los que se podría esperar la nivelación, en caso de haber coexistido protodorio y protojónicoático y protoarcadio en el Peloponeso micénico. Para el reparto de la conjunción condicional y de la partícula modal, si se siguen los esquemas de liorbes 1958 y Palmer 1962 , se explica mal que, conviviendo ya protodorios con protojonios y protoarcadios, fuesen radicalmente opuestas las respectivas elecciones; si se acepta que las formas de la conjunción y de la partícula fueron distintas e individuadas desde fecha antigua, la divergencia tiene todavía menos explicación: aquí y en lo que antecede seguimos ejemplificando con elementos centrales y frecuentes del sistema. Para repartos como el del aoristo en $-\xi \alpha$ o en $-(\sigma) \sigma \alpha$ de los verbos en $-3 \omega$ habrá que replantear la cuestión con un inventario detallado de formas: el de las délficas (cf. Moralejo I973b, pp. 217-223) no confirma la teoría tradicional, al menos la que se expone en Risch I955, p. 73 y en Ruijgh I96r, p. 2 Io.

II. Al lado de estas divergencias de fecha antigua que no fueron eliminadas o que surgieron pese a la presunta convivencia de dorios y no dorios, tenemos otras que nos presentan al dialecto de aquellos no participando en evoluciones del micénico, del protoarcadio y del protojonio, o realizando evoluciones que estos tres no conocen:

a) Insistimos en que la isoglosa $l i>s i$ es el eje central de la Dialectología griega; no es una forma de hablar o un latiguillo tradicional. al estilo de centum/satem para los indoeuropeistas. En nuestro caso la isoglosa se acompaña de otras coincidentes y de peso específico grande, además de completarse satisfactoriamente con lo que es ya y todavía conclusión válida de arqueólogos e historiadores.

b) Júnico-ático y arcadio producen la innovación oi, đi, mientras el griego occidental mantiene roi, $\tau \alpha$; si las formas cretenses y cirenaicas oi, ai son debidas al sustrato predorio, la fecha de la innovación ha de ser micénica o muy poco posterior a c. Ixoo, con lo que tenemos una nueva y significativa divergencia entre los presuntos conviventes. 
Casi podría decirse que, admitida la convivencia y admitida la correspondiente isoglosa, resulta más que sospechoso que el movimiento de nivelación e influjo mutuo entre ambos dialectos sea prácticamente nulo, 1o que está en contradicción con otras experiencias, por ejemplo la de la Inglaterra medieval, la de la Galicia actual, la de Grecia...

$\mathrm{Y}$ cerramos este apartado haciendo ver que entre dorio $\mathrm{y}$ jónicoático hay afinidades que no prueban nada a efectos de la tesis de Chadwick: la tendencia al timbre $a$ en la evolución de las sonantes; la sus-

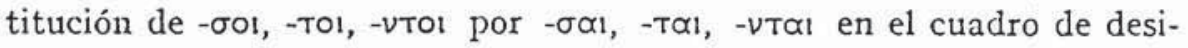

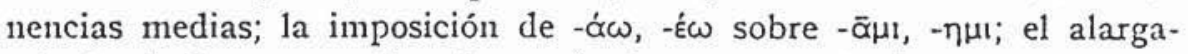
miento de $\dot{\varepsilon} v$ con -5 ; los alarganientos compensatorios, etc.: se trata de procesos comunes $y / 0$ postmicénicos a los que se sustraen ocasionalmente dialectos marginales (arcadio, eolio, noroccidentales). Y tampoco significan nada a favor o en contra de nuestra posición divergencias como $\bar{\alpha}>\eta$ en jónico-ático; distintos resultados en la contracción de vocales; apócope de preposiciones; etc.

I2. Iil problema de Creta es especialmente complicado y atrayente. lis evidente que en Cnoso hay griegos ya c. $1450 \mathrm{y}$, si se admite la fecha de c. 1400 para la destrucción del palacio y datación de las tablillas, se nos plantea un grave problema con el hecho de que el palacio ya no haya sido reconstruido y rehabitado. Otra cuestión es la de ¿quiénes fueron los destructores - los cretenses someticlos, los griegos mismos en discordia civil, un tercer pueblo? - : la propuesta por Chadivick de situar "a Doric-speaking population" en Creta y asignarle el papel de "lower classes" destructoras del palacio se apoya en que tampoco en Creta hay un cambio de cultura que atestigüe el de población y el de lengua; anteriormente hicimos nuestros reparos a este tipo de argumentación aplicada al caso griego, y añadimos que no es pequeño el cambio cultural que significa la total modificación de la organización política y económica de la isla, que, tras la caída de Cnoso, parece haber tenido la misma organización política que en el Peloponeso siguió a la caída de Pilo y Micenas.

Por otra parte, las huellas de sustrato que se registran en el dorio de Creta no las consideramos argumento que refuerce la tesis de Chadwick: son huellas perfectanente comprensibles aunque no hayan sido los protodorios —lower class»- quienes destruyeron Cnoso por propia iniciativa o aprovechando el impulso de "a force coming from outside Greece itselfn; es decir, aquí el problema es el mismo que para el sustrato predorio del Peloponeso, fueran o no los dorios quienes asaltaron e incendiaron Pilo y Micenas. 
Pero no deja de ser cliocaute la temprana ruina de Ciroso y que los dorios, ausentes de la épica, sean nombrados precisamente al hablar de Creta (Ot. NIX 17\%) y como opuestos a eteocretenses y a aqueus: sin duda habrá que admitir una conquista $y$ un asentamiento temprano de los dorios en (parte de) esta isla, aunque siga sub intice que hayan sido ellos los destructores cle Cnoso, y más todavía como clase dominada y sublevada.

13. En conclusión, tenemos muy serias dudas sobre la viabilidad de la tesis de Chadwick; a la escasa fuerza de los argumentos de orden arqueológico se une la imposibilidad para nosotros de admitir que protojonios y protoarcadios (es decir, micénicos) hayan convivido en el Peloponeso y antes de c. I200-Iroo con los protodorios sin que tal convivencia tenga su reflejo en la eliminación de divergencias esenciales y en la participación en canbios o innovaciones también esenciales y fechables en la época de tal presunta convivencia. Creemos que las diferencias entre micénico "normal" y "especial» están muy lejos de concluir la existencia de dos dialectos, e incluso dos niveles de lengua; creemos que en el mịcénico "especial" hay poco o nada que sirva a la "Dialektgliederung", y nada que no sea griego común, nada que sea específicamente dorio o protodorio. Entendemos, por consiguiente, que sigue siendo lícito y válido hablar de migración doria, pero entendida como movimiento dentro de un continuum espacial que, por lo menos desde c. 1900, es lingüisticamente griego. Para feclia micénica y afectando a la situación dialectal del primer milenio, la separación entre dorios, de un lado, $y$ micénicos, protojonios y protoarcadios, del otro, sigue siendo una realidad total (cf. Bartoněk I973).

I4. Coucluído el examen de la tesis de Chadwick, pasamos a sumarizar las coordenadas principales dentro de las que entendemos el problema de las migraciones y de los dialectos. Es muy de lamentar para nosotros que la posición de Chadivick resulte inviable, porque, de ser válida, hubiese abierto para nuestra disciplina un período de pax augustea comparable al que se dio cuando Hesíodo fr. 9 Merkelbacli-West (7 Rzach) parecía confirmado por la Lingüística y la Arqueología. Es claro que, si resultara cierta la tesis de Chadwick y 'Tebas u Orcómeno nos pusieran ante tablillas con eolismos evidentes, nuestra Dialectología iba a ser un rosario de seguridades salpicado de dudas de menor cuantía. Ėn la exposición que vamos a hacer el lector sabrá distinguir lo que es dominio público y común de lo que es posición de escuela o no pasa de opinión personal. 
La Dialectología del griego antiguo se ha ido despegando - aunque no totalmente, pues no sería posible- del esquema que equiparaba estirpe, dialecto y migración, con su natural secuela de que la mixtura dialectal luubiese de interpretarse como resultado de la de estirpes -además del lógico recurso a la "Wellentheorie». Aunque siga siendo válido, y sobre todo útil, hablar de dialectos dorio, jonio..., es evidente que las afinidades y las divergencias pueden tener poco o nada que ver con la división en estirpes, a las que el dialectólogo ha de agrupar en las formas más variadas y hasta contradictorias, además de escindir una nisma estirpe en dos o más ámbitos dialectales, incluso ya siu computar las variantes puramente locales; a esta nueva visión del problema. responde la nueva terminología, desde el ya veterano griego occidental, meridional..., a los "Saronischen Dialekte" que postula Bartonĕk 197r. (Para la historia reciente de la Dialectología griega nos remitimos a Chadivick r956, Cowgill r966, López Eire r968, Bartonĕk I972a; muy breve,-Radke 1967; Weiler 1976 ignora totalmente las aportaciones de Adrados, Garcia Ramón, López Eire, Ruipérez, Tovar; cosa tan impensable como callar aquí las de Heubeck, Hampl...: para manejar bibliografía sobre griego antiguo ya no está de más saber español.)

15. La quiebra de la ecuación de estirpe, dialecto y migración se ha producido en el terreno de la investigación arqueológica, en el de la lingüística y en el que intenta conjugar ambas; hoy parece imposible e inútil postular tantas migraciones como troncos dialectales -incluso haciendo reducción de éstos, por ejemiplo la de aqueo $=$ eolio + arcadiochipriota; tampoco parece posible o útil, si se admiten dos o más migraciones, dar nombre a cada una, si no es el genérico de no-doria vs. cloria; bautizar las migraciones con nombres concretos es lingüísticamente superfluo y puede confundir la realidad; arqueológicamente es cuestión problemática, aunque sean francamente atractivas tesis como la de Hammond 1972 , a la que tal vez empezariamos por discutir que los enterramientos de Micenas hagan cleducir que en ella vivieron "Aeolicspeakers", cosa que no aceptarán la mayoría de los micenólogos y dialectólogos.

Sobre todo interesa destacar que la Dialectología concluye hoy que la fragmentación del griego antiguo es, en su mayor parte, de fecha postmicénica, posterior al "movimiento" dorio, aunque ya en fecha micénica haya evoluciones importantes que permiten trazar isoglosas definitorias e incluso nos encontremos con diferencias remontables a época indoeuropea. 
I6. También se pone en cuestión la ecuación de lengua, pueblo y cultura a la hora de concluir asociación neccsaria entre esos conceptos y postular que el cambio de uno de ellos es deducible sin más porque haya cambio en los otros, y, a la inversa, el cambio en uno implinuc cambio en los demás; esto afecta muy íntimamente a lo que hemos pretendido páginas atrás, pues creemos que hay población y lengua nueva - dorios- pero no cultura nueva en el Peloponeso postmicénico. (Cf. Crossland y Birchall 1973, en especial Crossland en pp. 5-15, Blackman, Marinatos y Powell en pp. 315-319, Crossland y Birchall en pp. 323-3+7).

17. Cualquier esquenla teórico que se trace para explicar la población y las lenguas de la Hélade prehistórica tendrá sus argumentos y podrá encontrar paralelos que los apoyen, pero para la helenización de la Hélade -y perdónese tan oscura redundancia - parece lo más aceptable entender que:

a) Se excluye por sí misma la hipótesis de que la lengua griega sea autóctona, haya llegado a la Hélade por difusión o irradiación, forme parte de algún tipo de "liga" de lenguas más o menos afines en su gramática y vocabulario, pero 110 emparentadas históricamente y reducibles a una "Grundsprache»: la griega es una lengua indoeuropea que entra en la Hélade con la invasión de ésta por un pueblo indoeuropeo; el "indigenous development" de Renfrew I973 no parece muy viable.

b) Se impone, decinos, pensar en migración o invasión de indoeuropeos cuya lengua se define, por ejemplo, en los términos en que lo hace Wyatt 1970 - oclusivas sordas aspiradas, oposición de cantidad en las vocales, disimilación de aspiradas..., sufijo -Eús..., aumento en los tiempos verbales secundarios, etc.-; tal migración hubo de ser numerosa (no es viable la posición reciente de Schlerath x973, p. 9) y en algún aspecto - quizá capacidad guerrera y de organización política- superior a la(s) cultura(s) con que se encontró: ambos requisitos parecen necesarios para explicar que la lengua griega se impusiera a la pregriegas - más de una, desde luego, indoeuropeas y no indoeuropeas, por problemático que resulte el detalle, en el que no es momento de entrar-; de la superioridad cultural pregriega da cuenta el elevadísimo número de voces de tal procedencia que los inmigrantes incorporaron a su lengua, ya sin contar topo- y antroponimia.

c) Que la migración procede de los Balcanes nos parece fuera de duda (cf. Schachermeyr r954 y 1972, Hammond 1973, Crossland 1972, Georgiev $\mathbf{1 9 7 2}$, etc.) y excluido el itinerario que hace intervenir Anatolia como etapa desde o hacia los Balcanes, o desde el Mar Negro y el Cáucaso (cf. Hampl ig6o, Mellaart ig62, etc.). 
d) Mientras no se prueben mejor las alternativas propuestas, parece que las fechas de c. 2200 (Heládico Temprano III) y c. I900 (Heládico Medio I) son las más aptas para situar la nigración por lo que significan en cambios detectables por el arqueólogo (para la primera fecha, cf. Chadwick rg63; véase la reserva con que se expresa Caskey r964 frente a r969; para c. I9oo, cf. Haley y Blegen r928, Schachermeyr r954 y 1972, Wace 1956 y r962, Dow r960, Vermeule rg64). No parece imposible que ambas fechas puedan conciliarse, entendidas como momentos de un único proceso, y tienen ya en comù 1 que los arqueólogos coincidan en que significan la indoeuropeización de la Hélade; Schachermeyr r954 admite ta posibilidad de que ya antes de c. rgon uirgendwelche Vortrupps der urgriechischen Wanderungsgruppe in Hellas aufgetreten sein könnten.

Para la fecha c. I60o (Heládico Medio II) no parece haber argumentos arqueológicos suficientes y el lingüístico suele reducirse al poco probatorio "ex silention; no consigue convencernos Wyatt I970 con la argumentación sobre el carro y su léxico; contra toda evidencia está Hood r972, que retrasa a c. I200 la presencia griega en el Peloponeso, y tampoco estamos de acuerdo con el esquema de Hampl ig6o.

18. Hasta aquí lo referente a la migración, no migraciones, griega, ya que no hay migración, sino movimiento de dorios y noroccidentales. Intentaremos razonar cómo y por qué hablar de movimiento y no de migración para los griegos occidentales no es un simple ardid semántico.

Entendemos que lay una sola migración griega que, procedente de más al Norte, cubre toda la Hélade - en un primer momento ya, o progresivamente- descle Lipiro, Atamania, Macedonia y Tesalia hasta Mesenia y Laconia. Arqueológicamente no parece haber reparo invalidante: no hay fractura definitoria y está bien documentada la continuidad y relación entre Norte y Sur, aunque con total superioridad cultural de este último, en especial desde c. 1600.

Se ha razonado y ya es opinión común que la fragmentación dialectal no puede venir dada por la separación de estirpes - unas dentro y otras fuera de la Hélade- que se reencontrarian al final de las sucesivas y respectivas migraciones. Además de haberse constatado que el grueso de tal fragmentación es reciente o postnicénico, está el argumento cierto de que la separación de estirpes por espacio de hasta ocho y más siglos tendría que conducir a la cliversificación de la lengua, más que a su fragmentación dialectal, lo que choca frontalmente con la realidad del griego común, al que tampoco se hubiera llegado por el reencuentro de las estirpes. Fn consecuencia, parece necesario admitir 
que desde la separación del tronco indoeuropeo y a lo largo de la Edad del Bronce las estirpes griegas han estado en contacto continuo (o, a lo sumo, con pérdidas esporádicas y no duraderas de dicho contacto) y que, desde que ponen pie en la Hélade, esta es un continuım lingüístico griego para el que podemos suponer la siguiente distribución:

19. Son autóctonos en el sentido de que probablemente no han cambiado su emplazamiento primero, ni han sido desplazados de él por otras estirpes, y ni siquiera se han mezclado en proporción notable con gentes de esas otras estirpes - los áticos y los arcadio-chipriotas; es probable que otro tanto ocurra con epirotas, acarnanios, etolios, focidios y locrios, para todos los cuales puede suponerse un emplazamiento más septentrional antes del movimiento dorio; de los tesalios sabemos (Thuc. I 12,3) que desde la región del Pindo hubieron de moverse hacia la de Arne, y de ésta hacia el Este; su movimiento hacia Arne enipujó a los beocios - sin duda procedentes de la región del Boiov - hacia su emplazamiento de época histórica. No se excluye que la presencia eolia en 'Tesalia y en la Cadmeida sea ya anterior a estos movimientos.

Al contrario, el movimiento dorio desplaza o se impone a poblaciones protojonias y/o protoarcadias - micénicas en definitiva y sea cual sea la relación de ellas y su dialecto con la lengua de las tablillasen Acaya, Elide, Mesenia, Laconia, Argólida y Megárida. A la espera de lo que en su día puedan decidir nuevas tablillas de Tebas, podemos suponer que los beocios desplazan $y / 0$ se superponen a población jonia y eolia, parte de la cual parece haber emigrado al Asia Menor e islas próximas, si damos crédito a las tradiciones, en especial a las listas de metrópolis (cf., por ejemplo, Sakellariou 1958, pp. 239-243 y Cook 1964, pp. 4-17); que también Tesalia, Atica y el Peloponeso son punto de partida de migraciones al Asia Menor está fuera de duda, como también que el primer motor de éstas es el colapso micénico y subsiguiente presencia de griegos occidentales.

20. Para imaginar el contacto de protoeolios (futuros eolios de Lesbos y Asia Menor) con protojonios en la Cadmeida micénica, o, si se prefiere, contacto de esos protoeolios de la Cadmeida con protojonios del Atica, me baso en que, frente a beocio y tesalio, el lesbio conoce la asibilación $\mathrm{TI}>\sigma_{l}$ y alguna que otra afinidad importante con el jónico-ático. Tal proceso fonético es anterior a c. I 400 porque se documenta ya er: Cnoso, y en tal fecha es francamente impensable un contacto de protojonios $y$ protoeolios en Asia Nenor; por otra parte, no creo 


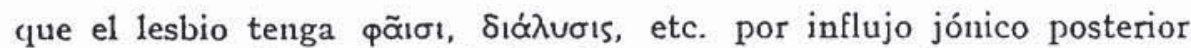
a la consumación del cambio fonético, porque no veo las razones y las vias para tal influjo, y sí las veo para otros de orlen morfológico y léxico, y de ahí que me abstenga de discutirlos aquí; prefiero inclinarme a creer que la isoglosa alcanzó a protoeolios limítrofes o conviventes con protojonios, cosa que solamente pudo haber ocurrido en la Cadmeida (y tal vez en Magnesia y Acaya Fitiótide por su contacto con Eubea). Mi posición es distinta, bien se ve, a la opinión tradicional y dominante desde los trabajos de Porzig y Risch, a los que aquí y ahora discuto únicamente la isoglosa $\mathrm{Tl}>\sigma \mathrm{l}$, y no la de mpós frente a moti, oi frente a тoi, etc. Al disponerme a rehilvanar estas páginas compruebo, y me felicito por ello, que en Adrados 1976, p. 255, se apunta una posición similar a la mía.

2I. Si el cuadre que trazamos es válido y el lector tiene a bien disculpar el esquematismo simplificador con que escribimos, para la interrelación de dialectos pudiera concluirse que:

a) Nunca hubo eolios al Sur de Beocia, ni jonios al Norte de ella, en fechas micénica y postmicénica. Los únicos contactos directos de jonios y eolios en fecha micénica son los del Atica y Eubea con la Cadmeida, Magnesia y Acaya Ftiótide; o en la Cadmeida entre jonios y eolios conviventes; o, si la Cadmeida era jonia, de jonios con eolios establecidos al Norte de ella.

b) Los contactos de estirpes y correspondientes dialectos en fecha micénica son fundamentalmente de eolios con griegos occidentales (iy bien occidentales eran en origen los Alo $\lambda$ Eis para Tucidides y $\mathrm{He}$ ródoto!), de un lado, y de jonios con arcadios, del otro.

c) Parece indefendible la hipótesis aquea y no hay contacto directo de arcadios con eolios; en líneas generales, la comunidad de eolio y arcadio está en conservar lo antiguo, y las afinidades recientes sou o coincidencias o partes de procesos comunes a todo el griego. L̇̀ cambio, sí parece justificado en líneas generales ver el eolio como puente entre griego occidental y jónico-ático.

d) El movimiento dorio pone en contacto estrecho a jonios y áticos con dorios; separa a jonios y áticos de arcadios.

c) En líneas generales, será válido el recurso al sustrato en el estudio del dorio y hablas noroccidentales del Peloponeso; en cambio, no será necesario ni quizá útil - salvo en algún punto concreto- para explicar el panorama complejo de la Grecia central, e incluso para el tesalio occidental: aquí seguirá teniendo vigencia parcial el recurso a la mixtura de poblaciones, siempre secundario frente al estudio que parte 
del hecho concreto, define su cronologia y reconoce su ámbito de difusión, todo ello con independencia de cuáles sean las estirpes o.poblaciones afectadas.

22. Con lo dicho venimos a coincidir, creemos, con esquemas como el bien conocido de Cowgill IgGG; con toclos los dialectólogos que han negado el grupo aqueo; con quienes ponen en entredicho la teoría del sustrato eolio en griego noroccidental (por ejemplo, García Ramón r973); también con quienes - Adrados, Heubeck, Risch, Ruijgh, 'Tovar, Cliadwick, Porzig, etc. (a la bibliografía indicada en el punto $x_{4}$ añádase Hiller y Panagl I9;6) - ponen en relación la lengua de las tablillas con arcadio-chipriota y/o jónico-ático, sea o no el micénico un dialecto sin descendencia directa, y hacen ver las afinidades fundamentales y las divergencias recientes entre arcadio-chipriota y jónico-ático, así como las divergencias originarias y las afinidades recientes entre jónico-ático y dorio. No concordamos con la opinión minoritaria (Gallavotti I958) que relaciona micénico y eolio, y no entramos en si el micénico era o no era una koiné, tesis hoy tan indemostrable como inatacable, pero errónea desde luego en pretender que tal koiné se continúe de algún modo en la lengua homérica (Georgiev 1972b).

Juan J. Moraliejo Alvarez

Universidad de Santiago

\section{BIBLIOGRAIIA}

Acta Myc. II: Acta Mycenaea. Proceedings of the Fifth Inlernational Colloquium on Mycenaean Studies... (1970), ed. M. S. Ruipérez. Vol. II, Communications (= Minos N. S., 197I). Salamanca, 1972.

Acta Aeg. Pr.: Acla of the Second International Colloquism on Aegean Prehistory. Athens, 1972.

ATrr Ronu: Alti e Memorie del r. ${ }^{\circ}$ Congresso Internazionale di Micenologia, Roma, 1967. Vol. XXV 1, 2, 3 de Incunabula Graeca. Roma, Edizioni dell'Ateneo, 1968.

Crossland y Burchan.s: Bronze Age Migrations in the Aegean... Proceedings of the First International Colloquium on Aegean Prehistory, Sheffield, ... Ed. R. A. Crossland and A. Birchall. Londres, Duckwortl, 1973.

I.BH KIRK: Language \& Background of Homer, ed. G. S. Kirk. Cambridge, Heffer \& Sons, 1964 .

ADRADOS I958: "La vocalización de las sonantes indoeuropeası, pp. 249-309 de FMIERITA 26. 
ADRADOS 1969: "Sobre la evoluciön griega de las sonantes indoeuropeas", pp. 63. 74 de Studia Classica et Orientalia Antonino Pagliaro oblata. Istituto di Glottologia della Università di Ronı. (Este trabajo y el anterior están reeditados en Eshbilios sobre las sonanles y laringales indoeuropeas, $2^{3} \mathrm{erl}$. Maclrid, CSIC, 1973.)

ADrunOS 1976: "Nicénico, dialectos paramicénicos y aqueo épico y "La creación de los dialectos griegos del primer milenios, pp. 66-I I 3 y $245-278$ de EMERIXA $44,1976$.

BAUER 1970: "De mycénien ALATOROPURO. AREPOZOO a grec MATPOחO-

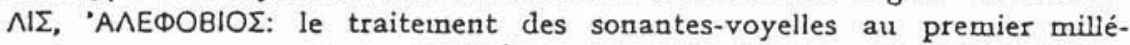
naire,, pp. 7-63 de Minos 8 .

BARTONĚK 1971: „Das Ostargolische in der räunlichen Gliederung Griechenlands*, pp. I1 8-122 de Donum Indogermanicum. Festgabe fïr A. Scherer..., ed. SchmittBrandt, Carl Winter, Feidelberg.

BARTONĚK 1972a: Classification of the West Greek Dialects at the time about 350 B. C., Pralia.

BARTONĚK 1972b: "Relevance of the Linear B Linguistic Phenomena for the Classification of Mycenaeann, pp. 329-345 de Acta Myc. II.

BARTONEK 1973: The Place of the Dorians in the Late Helladic World, pp. 3053011 de Crossland y Birchall.

Bringston 1969: Griechische Geschichte, $4^{\text {s }}$ ed., Mrunich. C. H. Beck,

Blackilan, MLnrinatos, Powel,: Mligration in Explanation of Culture Changev, pp. 315-319 de Crossland y Birchall.

CARPENTER 1966: Discontinuily in Greeh Civilization. Cambridge, University Press,

CAskry 1960: "The Larly Helladic Period in the Argolid, pp. 285-303 de Hesperia 29.

CAskriy 1964: Greece, Crele and the Aegean Islands in the Early Bronze Agt. Cap. XXVI(a) de C. A. H., vol. I. Cambridge, University Press.

Cinabivick 1956: "The Greeks Dialects and the Greek Prehistory", pp. 38-50 de Greece \& Rome N. S. 3 (= 1). 10 -118 de I.BII Kirk).

CnADwick I963: The P'rehistory of the Greek Language. Cap. XXXIX de C. A. H., vol. II. Cambridge, University P'ress.

C() in6.1: Greek Selle'ment in the Eastern Aegean and Asia Minor. Cap. XXXVIII de C.. A. HI., vol. II.

CowGilL 1966: aAncient Greek Dialectology in the Light of Mycenaean", pp. 7795 de Ancient Indoeuropean Dialects, ed. Birnbaun and Pubvel, Berkeley and Los Angeles, University of California Press.

Crossland y Birchali: "Retrospect and Prospects", pp. 323-347 de Crossland y Birchall.

CRUSSIAND 1972: "Recent Re-appraisal of Evidence for the Chronology of the Differentiation of Indoeuropeann, PP. $46-55$ de Acta Aeg. Pr.

Dissorougit 1964: The Last Mycenaeans and their Successors. Oxford, Clarendon Press. (Cf. también iHistory and Archaelogy in the Last Century of the Mycenaean Agen, pp. 1073-I093 de Atti Roma.)

Disborougur y HAmmond r962: The End of the Mycenaean Civilization and the Dark Age. Cap. XXXVI de C. A. H., vol. II.

Dow 1960: "The Greeks in the Bronze Age*, Pp. I-34 de XI Congres International de Sciences Historiques ( $=$ pp. 139-173 de LBH Kirk). 
FURBES 1953: "The relation of the Particle $\alpha v$ with $k \varepsilon(v), k \alpha, k \alpha v *, p p .179-182$ de Glotta 38 .

GaLLAvortr 195S: "Il carattere eolico del greco suiceneo", pp. I13-133 de RFIC $30^{\circ}$.

Garcli Rusóx 1973: "El llamado sustrato élico: revisión critica», pp. 233-277 de Cuadernos de Filología Clásica 5.

GEORGIEV I972a: "I,a Grèce et les Indo-européens" $y$ "Die Semeinsankeit der Kulturen Südbulgarieus und Nordgriechenlands wälırend des Neolitlikwuns, Äneolithikums uud der Irrülıbronzezeit,, pp. 24-38 y 115-128 de Acta Aeg. Pr.

GEORGLEV 1972b: «Le traitement des sonantes vojelles indo-européennes et le problème du caractère de la langue mycénienme», pp. 361-379 de Acta Myc. II.

HALEY Y BLECEN 1928: "The Coming of the Greeks", pp. I4I-I54 de $A J A 32$.

HAMnOND 1972: "The Coming of the Indo-europeans to the Southwestern Balkans*, pp. $\mathrm{IO}_{4}^{-1} 12$ de $\mathrm{Acta} \mathrm{Aleg}$. Pr.

HAmsond 1973: Shulies in Greek Mistory. Oxford, Clarendon l'ress. (En especial caps. I-III.)

HAmph 1960: "Die Clıronologic der Linwanderung der griechisclıen Stäume und das Problew der Nationalitït ler Träger der mykenischen Kultur*, pp. 5786 de $M H^{2} 7$.

HEUBECK 196r: "Zur dialektologischen Einordnung des Mykenischen", pp. 159172 de Glotta 29.

HILLER y PANAGL 1976: Die früngriechischen Texle aus mylentischer Zeit. Darmstadt, Wiseuschaftliche Bucligesellschaft. (Cf. en especial cap. 8.)

Hoon 1972: Arguments for the Arrival of the First Nou-Dorian Greeks in Southern Greece c. 1200 B. C.\#, pp. 62-71 de Acta Aeg. Pr.

LEJEUNF I968: "L'assibilation de 9 devant 1 en mycénien", pp. 733-743 de $\mathrm{Alti}$ Rona (= pp. $225-235$ de Mémorires... $3^{\text {a }}$ S., Incunabula Graeca XIIII. Roma, Edizioni dell'Ateneo, 1972).

LóPEz ELRE 1968: "Panorama actual de la Dialectologia Griega*, pp. 287-305 de $E C \mathrm{iz}$.

LOPE 2 EIRE 1974: "Eiu busca de la situación dialectal del jónico-áticon, pp. 247278 de Simposio de Colonizaciones, Barceloua.

Miellahar 1962: Anatolia c. $4^{000-2300 ~ B . ~ C . ~ C a p . ~ X V I I I ~ d e ~ C . ~ A . ~ H ., ~ v o l . ~ I . ~}$

MORAI.Ejo r973a: "Sonantes y Griego wicénico", pp. 409-426 de EMERITA $4 \mathrm{r}$.

MORLEJO 1973V: Gramílica de las inscripciones délficas. Universidad, Servicio de Publicacioues, Sautiago.

MIORPURGo 1968: "The Treatment of " $?$ aud *! in Mycenaean aud Arcado-Cyprian", pp. $79 \mathrm{I}-8 \mathrm{I}_{4}$ de Alti Roma.

Paluer 1962: "The Language of Hourern, pp. 75-r88 de A Companion to Homer, ed. Wace and Stubbings. Londres, Macunillan.

PORZIG 1954: Die Gliedernng des indogermanischen Sprachgebiels, Carl Winter, Heidelberg.

RuDKE 1967: "Dialekte" en Kileine Pauly II, cols. 1567-1574.

RENFREIV 1973: "Problems in the General Correlation of Archaeological and Linguistic Strata in Prehistoric Greece: the Model of Autocthonous Origin*, pp. 263-279 de Crossland y Birchall.

RrScir 1955: •Die Gliederung der griechischen Dialebte in neuer Sicht", pp. $6 \mathrm{r}$ 76 de $M H$ I 2 (= pp. 90-105 de LBH Kirk). 
121scir 1966: "I.es diffèrences dialectales dans le mycénien», pp. Ijo-I57 de Proceedings of the Cambridge Colloquinm on Mycenaean Shudies, ed. Palmer and Chadwick. Cambriclge, University Press.

RurJgir I96r: "Le traitement des sonantes voyelles dans les dialectes grecs et la position du mycénien", pp. 193-216 de Mnemosyne 14.

SAKEI,IARIOU 1958: La migration grecque en Ionie. Institut lirançais, Athènes.

Scincinermfyr 1954: "Prähistorische Kulturen Griechenlands", cols. r350-1 548 (en especial 1489-1494) de RE: XXII, 2.

SCHACHERMFYr 1972: "Vor-indo-enropäische Substrate und indo-europäische Zuwanderungen in der griechisch-anatolischen Frülızeit», Pp. ro-rt de Acla Aeg. Pr.

Schirrarnti 1973: Die Indogermanen. Das Problem der Expansion eines T'olkes im Lichte seiner sozialen Struktur. Innshrücker Beiträge zur Sprachwissenscliaft, 8.

Scinvyzir J)GE: Dialectormm Graecarum Exempla Epigraphica Potiora. Leipzig, 1923 (reimp. Ilildesheim, Olms, 1960).

Vintris y Cindmick 1973: Documents in Mycenaean Greeh, 2. a ed. por J. Charlwick. Cambridge, University Press, (Cf. también p. 173 y ss. de Chadroick, The Mycenaean World. University Press, Cambridge, 1976.)

Vermeule r964: Greece in the Bronze Age. University Press, Chicago. Trad. española, Grecia en la Edad del Bronce. FCE, México, I971.

WACE 1956: Foreword a la r.s ed. de Ventris y Chadrick, Documents...

WACE 1962: "The Early Age of Greece*. Cap. XII (pp. 33r-36r) de A Companion to Homer, ed. Wace and Stubbings. Londres, Macmillan.

WEILIER 1976: Griechische Geschichte. Darmstadt, Wissenschaftliche Buchgesellschaft. (Cf. pp. I6-40.)

WуATT 1970: "The Indo-europeanization of Greece", pp. 89-I I I de Indoetropean an Indoeuropeans, ed. Cardona, Hoenigswald and Senn. Philadelphia, University of Pennsylvania Press.

NOTA.- - Al releer estas páginas en la corrección de pruebas, quisiera pedir que se tenga por puramente formal la velemencia en la argumentación. Mi intención real es hacer ver que la muy sugestiva tesis de Chadrick no tiene, en mi opinión, apoyos sólidos e incontestablles en la documentación lingüistica de que disponemos. Ė̀ unodo alguno pretendo que tal documentación excluya la reconsideración del problema del dialecto y la migración de los dorios. 